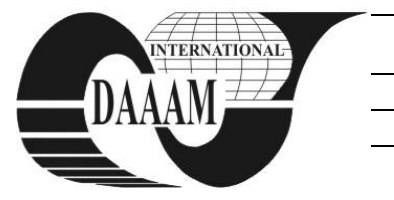

Annals of DAAAM for 2012 \& Proceedings of the 23rd International DAAAM Symposium, Volume 23, No.1, ISSN 2304-1382 ISBN 978-3-901509-91-9, CDROM version, Ed. B. Katalinic, Published by DAAAM International, Vienna, Austria, EU, 2012 Make Harmony between Technology and Nature, and Your Mind will Fly Free as a Bird

\title{
COMPUTATIONAL OPTIMIZATION OF VEHICLE AERODYNAMICS
}

\author{
AHMED, H[aseeb] \& CHACKO, S[ibi]
}

\begin{abstract}
Aerodynamics is the study of the effects of air when in motion. The vehicle aerodynamics have become crucial since have realized its importance. This study has been focused on the optimization of the aerodynamics with means of some external tools; an old fashioned car has been subjected and optimized. The tools used have been set to their optimum size, angle and shape to achieve the best. Next to reduction in the drag coefficient, lift coefficient has also been investigated and enhanced for better performance. The physical appearance of the car has been given keen significance while designing the external devices. All the analysis and modifications have been carried out computationally in the CFD software "ANSYS Fluent 14" and the CAD modeling in "Solid Works 2010".

Keywords: CFD analysis of car, computational optimization of car, aerodynamic tools designing, drag reduction of old car
\end{abstract}

\section{INTRODUCTION}

The fuel consumption of car has become crucial to think about as emission of $\mathrm{CO}_{2}$ in the environment has shown negative consequences. The study of aerodynamics involves the drag force that opposes the motion of a car, this have considerable contribution in the consumption of fuel. The coefficient of drag varies from car to car; hence manufacturers allot big proportion of their attention to aerodynamics.

There are many regions in the car that contributes in increasing the drag coefficient; in this study they all have been encountered. A number of aerodynamic tools are seen in the market today to decrease the impact and weaken those regions. In this study a number of devices have been designed and analyzed for decreasing the drag force.

The action of considerable down force is also advantageous when driving at high speeds. Some devices have been designed for achieving that. The down force increases the traction of the car hence bestowing stability. The down force is very significant when measuring the performance of a car, since the presence of this allows to corner at high speed.

Vortex generators and front bonnet duct are applied to contribute in reducing the drag coefficient while ground clearance, diffuser and rear wing for increasing the negative lift force. At the first drag coefficient has been considered followed by the negative lift coefficient.

\section{COMPUTER AIDED MODEL}

A taper, boxed shape car has been designed for the analysis and optimization. The model of the car has been designed using CAD software, Solid Works 2010, rendered image of the model is shown in Fig. 1.

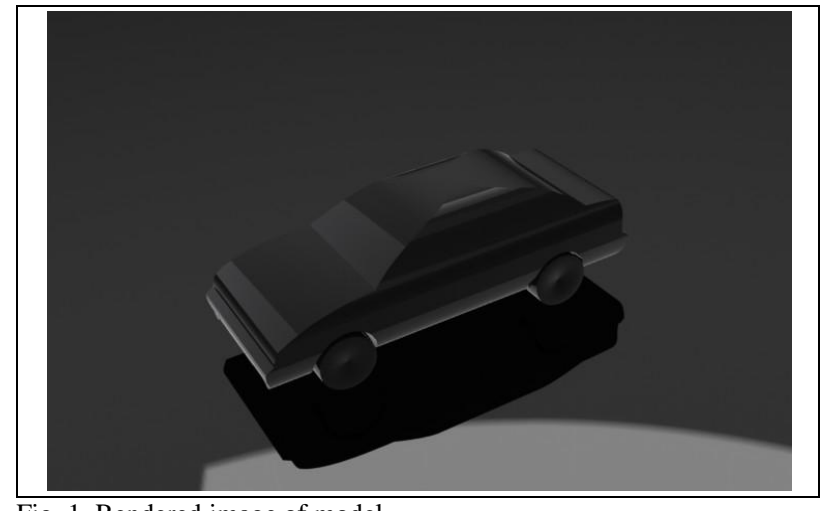

Fig. 1. Rendered image of model

\section{CFD SETUP}

Domain is a requirement for external flows analysis. It is a box filled with fluid and has boundaries. These boundaries are given conditions and the fluid is given a motion

The accurate result from CFD though is impossible but least error can be achieved with an appropriate size of domain. Considering this the suiTab. size used for this type of analysis is demonstrated pictorially in Fig. 2.

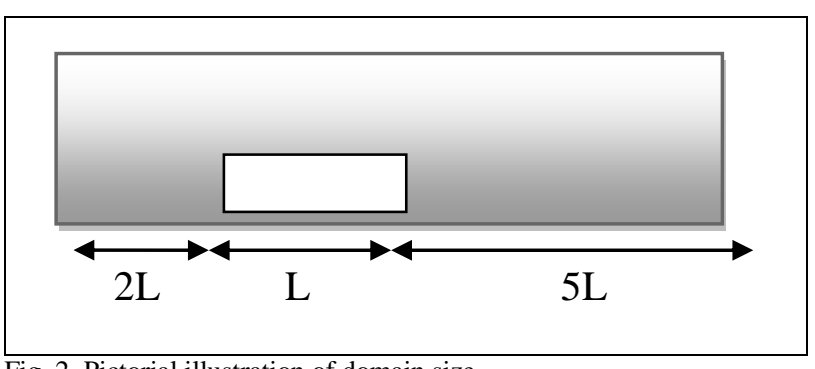

Fig. 2. Pictorial illustration of domain size

The height and the width of the domain were allotted 2 times the Length (2L).

On the other hand the boundary condition for the flow is also an important factor. Boundary conditions used for this analysis are given in Tab. 2 . 


\begin{tabular}{|c|c|}
\hline Velocity inlet & $20 \mathrm{~m} / \mathrm{sec}$ \\
\hline Sides and top & Symmetric walls \\
\hline Road & wall \\
\hline
\end{tabular}

Tab. 2. Boundary conditions

Once the simulation was achieved forces acting in the horizontal and vertical direction were calculated. The horizontal axis and the vertical axis represent drag and lift respectively. The coefficient reported for the individuals are shown in Tab. 3 .

\begin{tabular}{|c|c|}
\hline Drag coefficient & 0.4103 \\
\hline Lift coefficient & 0.2599 \\
\hline
\end{tabular}

Tab. 3. Coefficients for basic model

The positive sign for the drag represents the force in an opposite direction to the motion of the car whereas for the lift coefficient presents the vertically upward acting force.

\section{ANALYSIS OF BASIC MODEL}

The first approach for the optimization involves the detail study and analysis of the basic model. To read the flow and encounter the regions responsible for high drag is the main purpose of this step.

The analysis of the basic shape presents the value of drag coefficient as 0.4103 and the lift coefficient as 0.2599 . The coefficient of lift obtained gives an idea about the behavior of car at the speed of $20 \mathrm{~m} / \mathrm{sec}$. The air exerts the force on to the car that lifts the car of the ground assisting instability rather than pushing it into the ground. Primarly the drag coefficient has been targeted to be reduced.

The images below show the flow of air around the car and further shows the detailed analysis of the regions strengthening the drag coefficient.

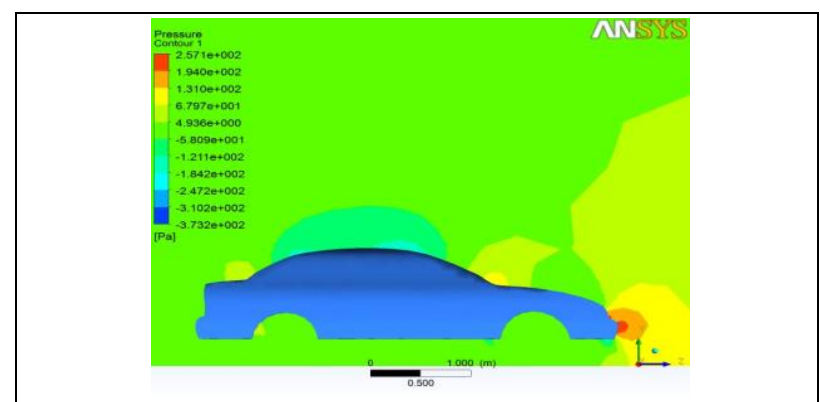

Fig. 3. Pressure contours across car

The front of a car stagnates the air hence increases the pressure; this causes the good proportion of high pressure region. At the inclined front windscreen due to sudden high angle the pressure also seems to be increasing to the level of yellow region that is consider as high pressure as well. Across the windscreen at the top the velocity increases again causing reduction in pressure. Finally at the rear declining angle the pressure increases due to flow separation occurring.
In order to decrease the drag coefficient these high regions are to be considered and optimized.

The air beneath the vehicle is of high pressure compared with the air above it. For introducing the negative lift force that plays a vital role in stability, the low pressure region has to be created underneath the car body.

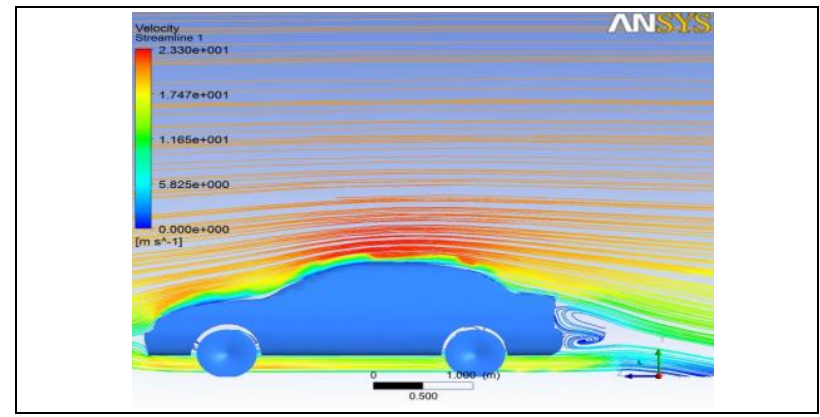

Fig. 4. Velocity stream lines around the car

Behavior of air streams shows the occurrence of an immense flow separation region behind the car. Vortices are generated because of pressure differences of the air exiting top of a car and the under the car. The flow separation and the production of vortices at the rear of the car also increases the drag coefficient hence has to be accounted.

\section{BACKGROUND THEORY}

Reducing the drag coefficient aims to reduce the area of flow separation, and also reduce the chance of air to stagnate. The first device installed for the purpose is vortex generators.

\subsection{Vortex generators}

Vortex generators are the devices that produce vortices in the air flow. These devices are stick at the top end just before the declining of rear wind screen of a car, to delay the flow separation at the rear.

The vortex generator follows the theory of exchange of momentum between upstream and downstream flow [1]. At the rear end due to taper shape the pressure of air at downstream increases compared to upstream and hence reverse force is generated that acts as opposing force.

With the installation of this tool the pressure gradient $\frac{d P}{d x}$ between the upstream and downstream is equalized and hence the opposing force is prevented. [1]

\subsection{Front Bonnet Duct}

There are multiple areas counted in high pressure regions around the car, these regions are to be cut down for the reduction in drag.

The high pressure regions are linked with speed of air, when the air speed reduces pressure increases, this pattern can be observed form Fig. 3 and 4 .

The front bonnet duct is a device that has a purpose of reducing the high pressure region at the start of front 
windscreen. Flow of air in this region is disturbed due to sudden increase in angle resulting in the air speed to reduce and pressure to increase. This device has to be designed such that the air is prevented from the reduction of velocity as much possible.

Vortex generators and front bonnet duct are reducing the drag coefficient, now down force is subjected to approach. This aerodynamic tools from generation of down force may increase the drag, hence has to be designed carefully.

\subsection{Ground Effect}

If air flows around the object and shape causes the formation of uncommon pressure regions, high pressure region exerts a force towards low pressure region.

The ground effect applies the Bernoulli principle, the area underneath the car is reduced so that the incoming air should experience reduction in area and must increase its speed gradually producing a low pressure region underneath. The high pressure region above the vehicle exerts the force towards the low pressure region, causes production of down force with the influence of oncoming air. [2]

\subsection{Diffuser}

Ground effect produces tremendous amount of down force, also increases the drag. The high speed air leaving the under body of a car at rear encourages the flow separation to widen. The pressure differences between upstream and downstream at the rear induce circulation in a flow. This circulation results in addition in drag force. To avoid such happenings a device known as diffuser is used

Diffuser is a design that is installed under the car at the rear and is considered as a part of underbody tray. This increases the area gradually at some angle and tends to bring air back to its original velocity at the time of exit. This practice prevents the formation of circulation in a flow. [3]

\subsection{Wing}

A wing is a tool that produces lift force in the airplanes. These wings can also be used in ground vehicle in inverted position to produces the negative lift force. Wings have become very famous as they have a tendency to produce good amount of down force with relative less amount of drag. [3] [4]

The wing works on Bernoulli principle to produce the down force by creating variant pressure regions around it. In the application of cars the low pressure region has to be under the wing and vice versa.

\section{DESIGN}

The designs of the tools are shown below individually in the headings below:

\subsection{Vortex Generators}

The designing of tools have been done considering the flow around the subjected vehicle as discussed in section 4. At first the drag coefficient is targeted hence vortex generators are designed at the first.

There are different shapes of vortex generator, delta shape vortex generator are most effective hence are used. [1]

The efficiency of the vortex generators depends on the design, height $(\mathrm{H})$, width and their numbers. The measurement of boundary layer thickness of the site is important because the most effective generators are the one which has its height approximately equal to the boundary layer thickness. Once the boundary layer thickness is known the base length can be $2 \mathrm{H}$ and the distance between adjacent two can be $100 \mathrm{~mm}$. [1]

The boundary layer thickness of the flow was calculated from plotting the graph of velocity Vs height. The boundary layer thickness can be obtained from the velocity distribution pattern

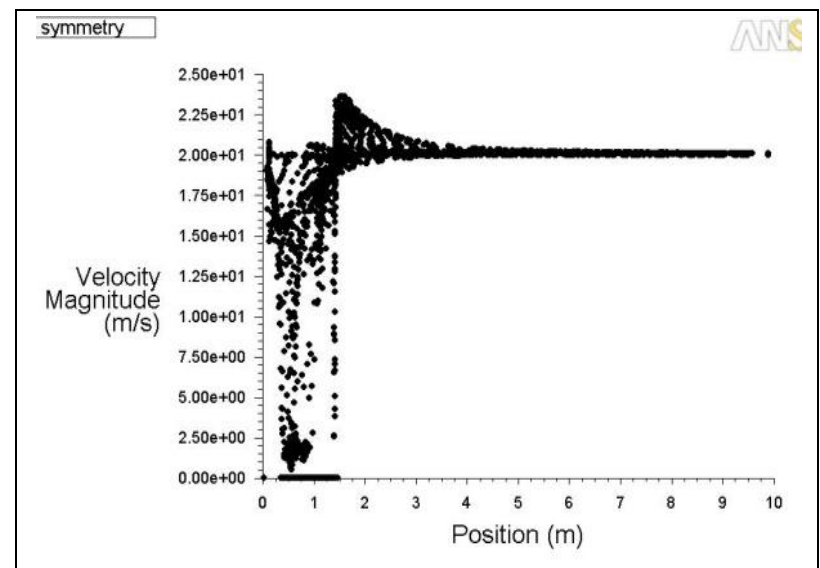

Fig. 5. Velocity Vs height graph for estimation of boundary layer thickness

The estimated value of boundary layer thickness from the graph is $0.02 \mathrm{~m}$. The design of the vortex generator is shown below:

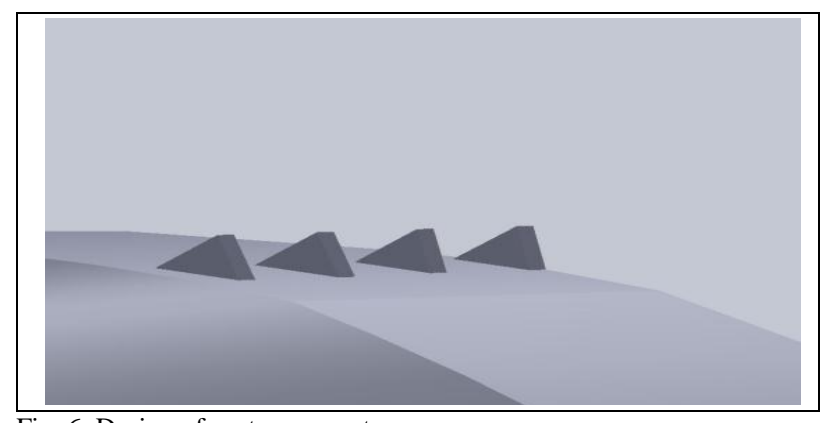

Fig. 6. Design of vortex generator

\subsection{Front Bonnet Duct}

Front bonnet duct is used for the purpose of increasing the air intake for an engine of a car. The design has been used in the other way around with elevation angle facing the front wind screen. The theory used for this modification is due to sharp angle of front windscreen the flow velocity at the front drops to zero that causing increase in the drag. 
This device direct almost half of the flow air at an angle that makes the flow not meet the area of front windscreen and the velocity of the flow doesn't stagnate.

The design of this device has to be such that it should be able to carry the approaching air smoothly onto it and then leaves it at a good angle such that it shouldn't be stagnated due to sharp angle of front wind screen. The design of the device is shown below fixed in the car bonnet.

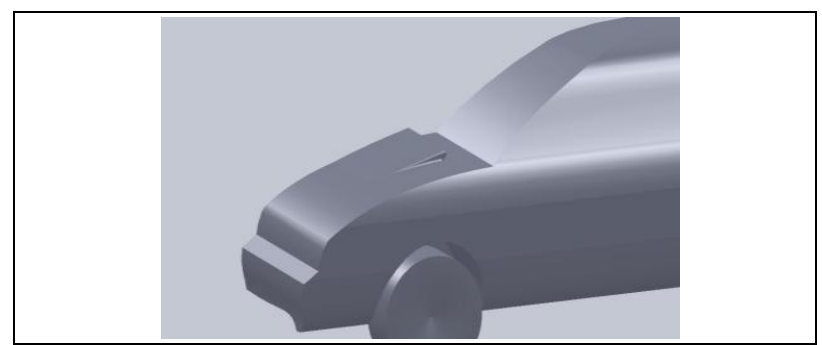

Fig. 7. Design front bonnet duct

\subsection{Ground effect and diffuser}

As discussed in the theory section that the area under the car has to be decreased for inducing ground effect. In an approach to reduce the area the ground clearance of car has been subjected to reduction.

Designing a diffuser depends on the angle of elevation and its start position. The effective elevation angle is the one that prevents the formation of the flow separation. The angle and the position have been finalized with an experience gained by studying variant shaped designs.

The requirement of high pressure region underneath the car could be obtained by reducing the ground clearance hence it has been reduced from $0.17 \mathrm{~m}$ to $0.08 \mathrm{~m}$. The Tab. below shows the specifications of the finalized design:

\begin{tabular}{|l|l|}
\hline Height & $0.08 \mathrm{~m}$ \\
\hline Diffuser angle & 3.45 degree \\
Diffuser start position & Half way \\
\hline
\end{tabular}

Tab. 3. Specifications of Diffuser

The image below shows the car equipped with reduced ground clearance and the diffuser that starts from the center of the car.

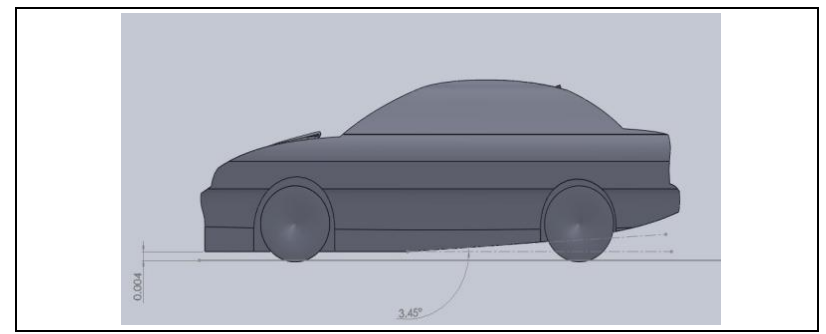

Fig. 8. Ground effect and diffuser

\subsection{Wing}

An airfoil or wing is a device that produces tremendous amount of down force and is very significant tool when down force is in demand. The presence of the wing also induces the drag coefficient.

A car wing produces the higher pressure region under itself. This induces a force acting vertically downwards.

Wing designed for a model is shown in Fig. 15

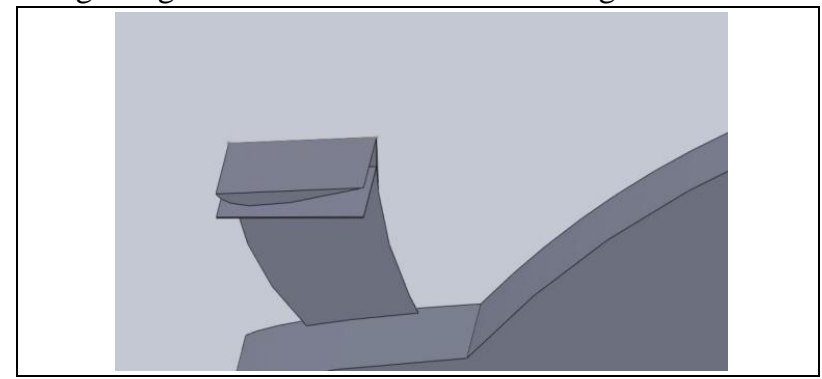

Fig. 9. Wing designs

It has been placed at good height so that it could meet less diverted stream of air, the thin plate has been designed to produce ground effect by inducing high pressure region under the wing 4.

The specifications of the design are presented in Tab.

\begin{tabular}{|l|l|}
\hline Chord length & $0.2 \mathrm{~m}$ \\
\hline Height form boot & $0.3 \mathrm{~m}$ \\
Angle & 0 degrees \\
\hline
\end{tabular}

Tab. 4. Specifications of wing

\section{RESULTS}

The results for each modification are presented in this section. The previous modification that resulted in noticeable improvement was included in the model. Further tool were analyzed on that model.

\subsection{Vortex generators:}

The results obtained with the device are presented in Tab. 5

\begin{tabular}{|c|c|}
\hline Drag coefficient & 0.39 \\
\hline Lift coefficient & 0.248 \\
\hline
\end{tabular}

Tab. 5. Results obtained from vortex generators.

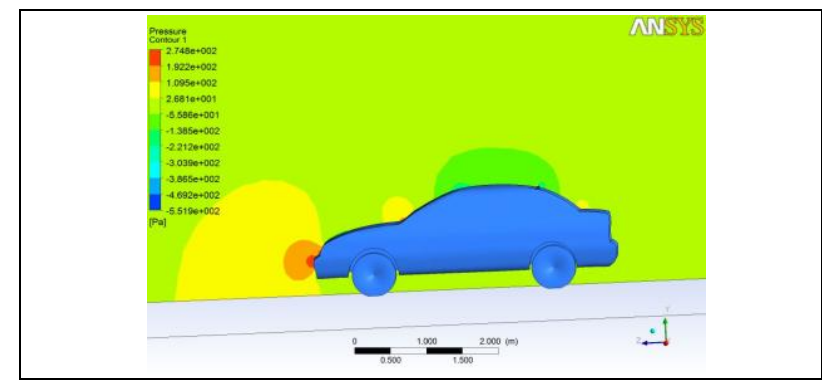

Fig. 10. Pressure contours of Vortex Generator design

The vortex generators by an exchange of momentum between upstream and downstream of the flow reduce the high pressure region at the rear wind screen; can be observed by comparing Fig.s 8 and 4 . 


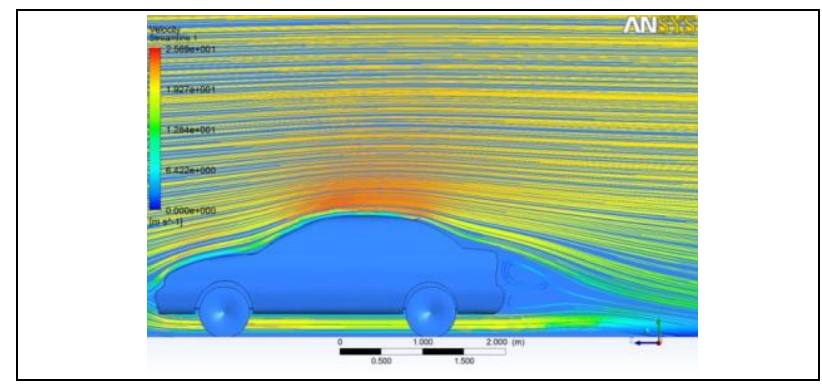

Fig. 11. Streamlines around of Vortex Generator design

The vortex generator besides reducing the drag also creates a drag because of its presence. The efficiency of the vortex generator is the measure of ratio between the drag generated to drag reduced by the generators. In the design shown in Fig. 8 the total drag is be reducing by a great extend hence they are considered to be performing well.

The coefficient of drag has been reduced from the value of 0.4103 to 0.39 .

\subsection{Front Bonnet Duct}

The results obtained from the design are shown in Tab. 6

\begin{tabular}{|c|c|}
\hline Drag coefficient & 0.377 \\
\hline Lift coefficient & 0.2944 \\
\hline
\end{tabular}

Tab. 6. Coefficients of front bonnet duct

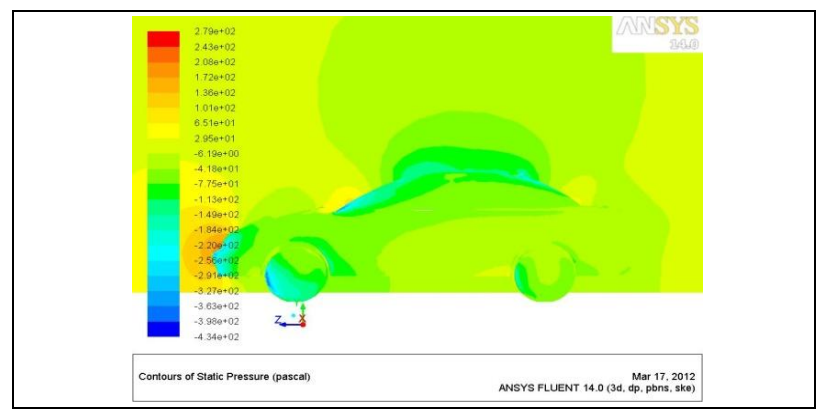

Fig. 12. Pressure contour of front bonnet duct

The high pressure region at the front seems to be reducing hence the drag coefficient as well, which can be observed comparing Fig.s 10 and 4.

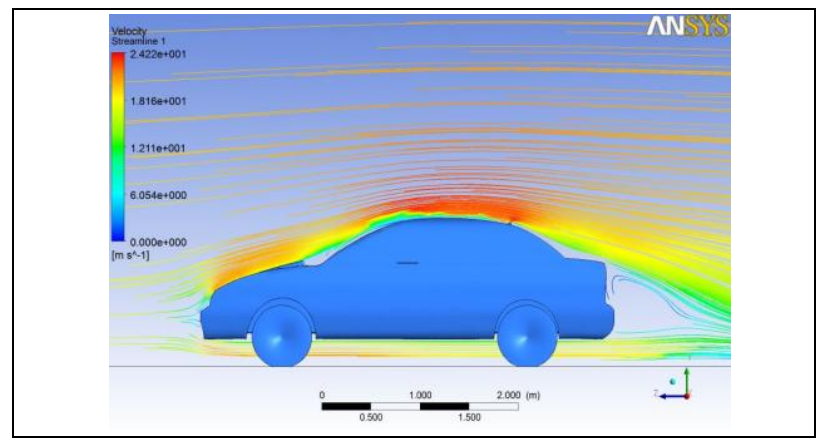

Fig. 13. Stream lines of front bonnet duct

The streamlines presented in the Fig. above shows the working of the tool. The device doesn't allow the air to lose its velocity unlike experiencing in the basic model. This reduces the high pressure region. The drag coefficient falls gradually from 0.39 to 0.377 with the aid of front bonnet duct.

\subsection{Ground effect and diffuser}

Earlier in this modification the drag coefficient was noted as 0.377 where as the lift coefficient 0.2944 . The positive value in the lift coefficient indicates the vertical upwards acting force, serving to lift the car rather than stick onto the ground. This kind of lift force often results instability in car at high speed.

After the ground effect and the diffuser the coefficient for the drag and the lift obtained are shown in the Tab. 7

\begin{tabular}{|c|c|}
\hline Drag coefficient & 0.3748 \\
\hline Lift coefficient & 0.094 \\
\hline
\end{tabular}

Tab. 7. Coefficients with ground effect and diffuser design

The ground effect drops the lift force that was reported form the basic model drastically to a very small value due to creation of high pressure region under the car. This higher pressure region can be observed from the pressure contour in Fig. 13.

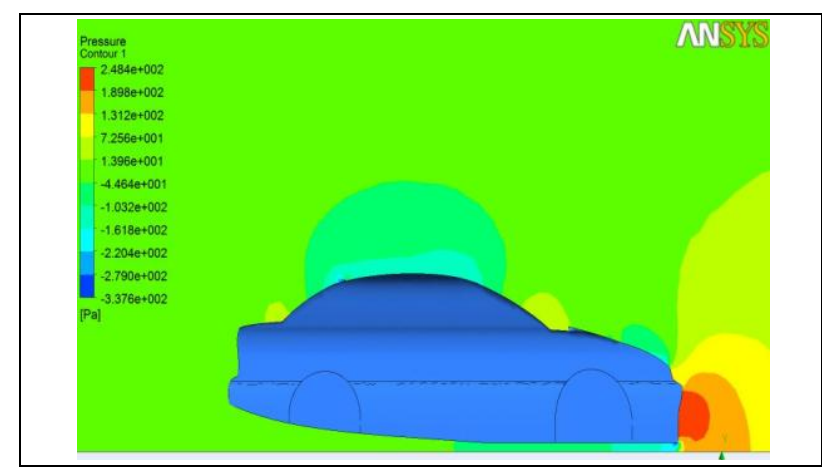

Fig. 14. Pressure contours of diffuser design

The first half under the car indicates the high pressure region compare to the rest half part where the diffuser is situated. At the rear of the car the pressure upstream and downstream seems to be equal hence the diffuser is working well.

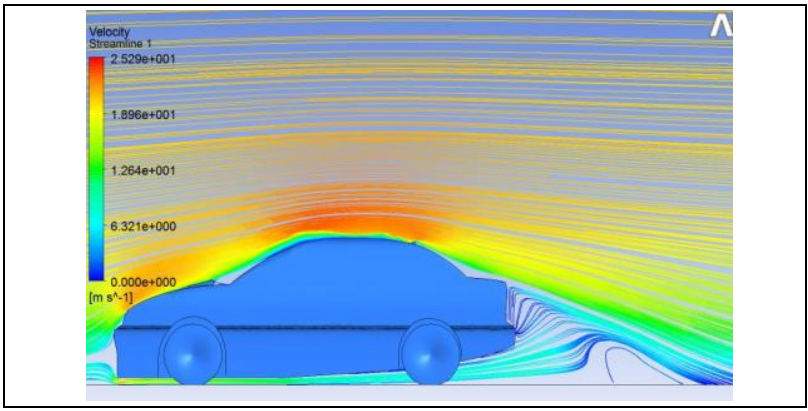

Fig. 15. streamlines of diffuser design

Fig. 14 indicates a relative less flow separation area and the circulation at the rear of a car. The Fig. also shows the magnitude and can be observed that the air velocity gradually drops when meets the diffuser.

The fine working of the diffuser design enables the drag coefficient to be maintained with a slight different 
value of 0.3748 where shows a big difference in lift coefficient, from 0.2944 to 0.094

\subsection{Wing}

The result obtained from the design installed with a wing is shown in Tab. 8.

\begin{tabular}{|c|c|}
\hline Drag coefficient & 0.395 \\
\hline Lift coefficient & 0.00659 \\
\hline
\end{tabular}

Tab. 8. Coefficient obtained after wing installation

The wings causes the drag coefficient to rise because of its presences, the lift force at the speed of the test has been almost reached to the value of zero. This shows that the wing produces the negative lift force but also induces good amount of drag.

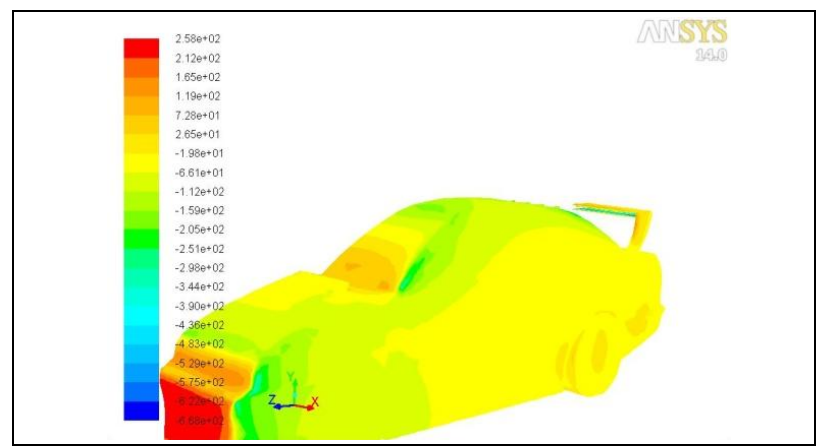

Fig. 16. Pressure contour with wing

The pressure contour in Fig. 16 shows that the front area of the wing and the plate is at high pressure, this can be marked as reason for rise in drag coefficient.

Model equipped with wing gradually increases the drag from 0.3748 to 0.395 but drops the lift coefficient from 0.094 to 0.00659 .

\section{CONCLUSION}

Efficient aerodynamics results in the improvement of many factors involved with the motion of a car such as fuel consumption and the performance. With the decrement in the drag coefficient the fuel consumption can be improved as there would be less opposing force acting on the car. As far as the performance is concerned the induction of downforce is the prime factor of the performance. When car would be stuck harder into the ground it would result in better performance.

These vital effects are produced with the aid of the devices designed in this study. When comparing the pressure contours of the before and after we experience marginal differences. The pressure changes around the car due to modification and the Fig. 18 depicts the changes. The comparison of the pressure contours in the before and after is presented in the Fig. below:

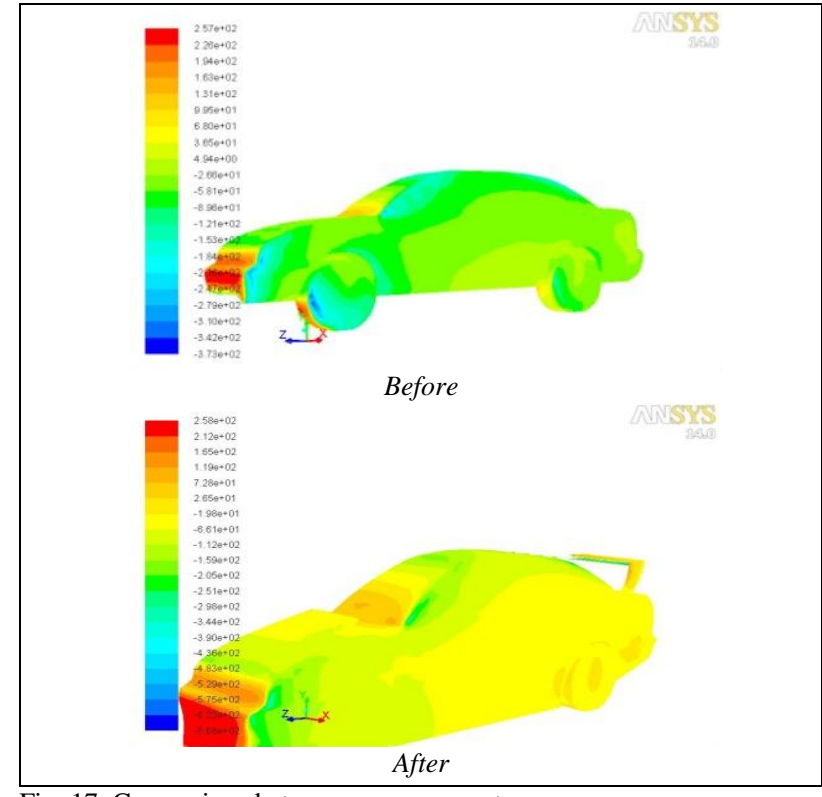

Fig. 17. Comparison between pressure contours

The velocity stream lines with presentation of velocity magnitude can also be compared; noticeable changes can be observed and these are the cause of better aerodynamics.

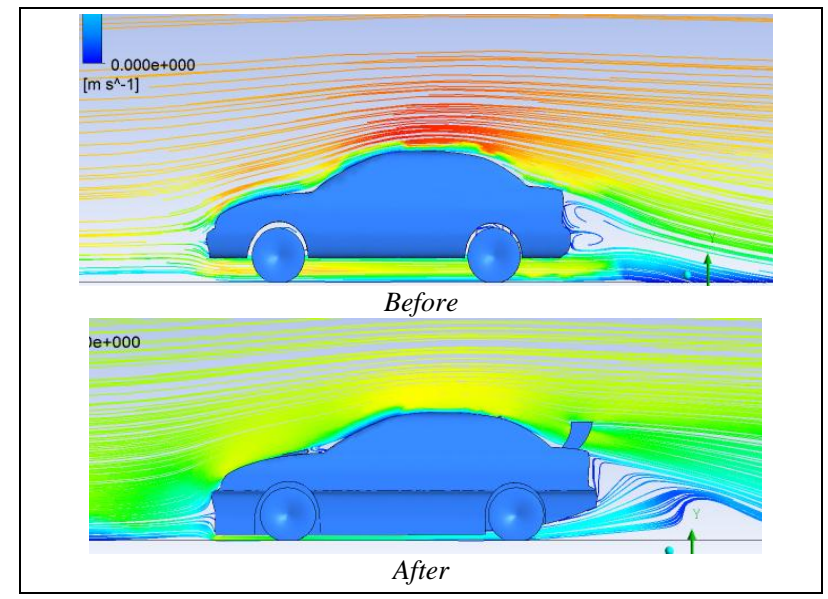

Fig. 18. Comparison between velocities streamlines

The area of flow separation seems to be reduced along with circulation of the flow. These factors have good influence in the reduction in the drag coefficient.

\section{REFERENCES}

[1] Masaru Koike; Tsunehisa Nagayoshi and Naoki Hamamoto. (2004) No. 16, Research on Aerodynamic Drag Reduction by Vortex Generators, technical papers

[2] Mohd Aliff Bin Mohd Nor and Aswatha Narayana, CFD Study of Rear Spoiler Fitted to Sedan Car, Technical report

[3] J. Katz (2006) 2nd edition, Race-Car Aerodynamics, Robert Bentley Inc., Cambridge, Massachusetts

[4] Debojyoti Mitra (2010) Vol. 2 (7), Design Optimization of Ground Clearance of Domestic Cars, International Journal of Engineering Science and Technology 